\title{
Éxito de la cirugía bariátrica: perspectiva biológica
}

\author{
Success of bariatric surgery: Biological perspective \\ Sucesso da cirurgia bariátrica: perspectiva biológica
}

Brenda K. Delgadillo Arteaga', Edna J. Nava-González²*.

Recibido: 26 de febrero de 2021. Aceptado para publicación: 16 de junio de 2021.

Publicado en línea: 28 de junio de 2021.

https://doi.org/10.35454/rncm.v4n4.286

\section{Resumen}

La obesidad se define como una acumulación anormal o excesiva de grasa corporal, que puede alcanzar grados capaces de afectar la salud. Se sabe que existe una correlación directa con el aumento del índice de masa corporal (IMC) y la prevalencia de comorbilidades, lo que genera un impacto negativo en la esperanza de vida y el gasto en salud pública. Entre todos los tratamientos para la obesidad disponibles, la cirugía bariátrica es la que presenta mejores resultados en la pérdida de exceso de peso y remisión de comorbilidades, y produce efectos positivos en la función del tejido adiposo, la sensibilidad a la insulina, el contenido y función de la grasa hepática, así como la mejoría en general del metabolismo y, en consecuencia, aumenta la calidad de vida a largo plazo. Las mejorías biológicas y metabólicas son posibles en pacientes con obesidad grave después de la cirugía bariátrica.

Palabras clave: cirugía bariátrica, metabolismo, balance energético.

\section{Summary}

Obesity is defined as an abnormal or excessive accumulation of body fat, which can reach degrees capable of affecting health. A direct correlation is known to exist between the increase of body mass index (BMI) and the prevalence of comorbidities, resulting in a negative impact on life expectancy and public health spending. Among all treatments currently available for obesity, bariatric surgery is the one with the best results in terms of excess weight loss and remission of comorbidities, and additional positive effects on adipose tissue function, insulin sensitivity, liver fat content and function, as well as overall improvement of metabolism and, consequently, increases in long-term quality of life. Biological and metabolic improvements are possible in severely obese patients after bariatric surgery.

Keywords: Bariatric Surgery; Metabolism; Energy Balance.

\section{Resumo}

A obesidade é definida como um acúmulo anormal ou excessivo de gordura corporal, que pode atingir níveis capazes de afetar a saúde. Sabe-se que existe uma correlação direta com o aumento do Índice de Massa Corporal (IMC) e a prevalência de comorbidades, gerando um impacto negativo na expectativa de vida e no gasto público em saúde. Entre todos os tratamentos disponíveis para a obesidade, a cirurgia bariátrica é a que apresenta melhores resultados na perda do excesso de peso e remissão das comorbidades, gerando efeitos positivos na função do tecido adiposo, na sensibilidade à insulina, no conteúdo e na função da gordura hepática, bem como na melhora geral do metabolismo e como consequência, aumentando a qualidade de vida em longo prazo. Melhorias biológicas e metabólicas são possíveis em pacientes com obesidade severa após a cirurgia bariátrica.

Palavras chave: cirurgia bariátrica, metabolismo, balanço energético.
Centro de investigación en Ciencias Médicas, Cuerpo Académico de Salud del Universitario, Universidad Autónoma del Estado de México, México.

2 Facultad de Salud Pública y Nutrición, Universidad Autónoma de Nuevo León. Monterrey, Nuevo León, México.

\section{INTRODUCCIÓN}

La cirugía bariátrica (CB) es el tratamiento para la obesidad mórbida (definida por un IMC $\geq 40 \mathrm{~kg} / \mathrm{m}^{2} \mathrm{o} \geq$
*Correspondencia: Edna J. Nava-González. edna.navag@uanl.mx

$35 \mathrm{~kg} / \mathrm{m}^{2}$ con una comorbilidad asociada) más efectiva a largo plazo, pero no está libre de complicaciones. La CB puede lograr una pérdida de peso sustancial y, a largo plazo, conduce a una mejoría o resolución 
de complicaciones, incluida la diabetes mellitus tipo 2 (DM2), la hipertensión, la enfermedad del hígado graso y la infertilidad asociada con la obesidad, así como una reducción de la mortalidad ${ }^{(1)}$.

Actualmente, los procedimientos que se realizan con más frecuencia son la derivación gástrica en $Y$ de Roux (BGYR) y la gastrectomía en manga o manga gástrica $(\mathrm{MG})^{(2-4)}$. Ambos procedimientos producen una pérdida de peso significativa y sostenida. Inicialmente, los procedimientos bariátricos estaban destinados a causar pérdida de peso mediante la restricción física de la ingesta de energía, como en la banda gástrica, o la reducción de la absorción de energía a través de la malabsorción, como en el cruce duodenal ${ }^{(5)}$; sin embargo, los cambios en la anatomía gastrointestinal tanto en BGYR como en MG dan como resultado varias adaptaciones fisiológicas que impulsan la pérdida de peso independientemente de la malabsorción y la restricción, por lo que esta clasificación ya no se aplica a la BGYR o la MG. Aunque aún no se entienden completamente, impulsan la pérdida de peso mediante cambios en la conducta alimentaria, lo que a su vez reduce la ingesta energética ${ }^{(4,5)}$.

Después de la $\mathrm{CB}$, además de aumentar la saciedad y reducir los niveles de hambre, se observa un cambio marcado en la elección de alimentos; las personas informan un cambio en la preferencia de alimentos que se aleja de los alimentos con alto contenido de azúcar y grasa, que comúnmente causan aversiones ${ }^{(6)}$. El objetivo de esta revisión es reconocer las mejorías biológicas y metabólicas que tienen los pacientes con obesidad grave después de la realización de la CB.

\section{BENEFICIOS METABÓLICOS}

En particular, la cirugía BGYR y la MG son los tratamientos para la obesidad más efectivos, con una pérdida de peso sostenida del $15 \%-25 \%$ hasta por 20 años $^{(7)}$. Para los pacientes con obesidad grave (IMC $\geq 40 \mathrm{~kg} / \mathrm{m}^{2} \mathrm{o} \geq 35 \mathrm{~kg} / \mathrm{m}^{2}$ ) y diabetes, los beneficios a largo plazo de una mayor esperanza de vida y calidad superan claramente las desventajas de que la cirugía sea invasiva e irreversible ${ }^{(4)}$.

Recientemente se demostró que la $\mathrm{CB}$ induce un nuevo estado metabólico que parece conservarse en roedores y humanos. Después de la CB, la inflamación crónica de bajo grado característica de la obesidad se resuelve y se mejora la liberación de hormonas del tipo incretinas ${ }^{(8,9)}$. Además, la oxidación de lípidos y la función mitocondrial mejoran después de la CB. Sin embargo, el mecanismo molecular detrás de esto aún no está claro ${ }^{(8)}$.
Días después de la intervención quirúrgica se presenta un aumento importante en la secreción de varias hormonas intestinales, incluido el péptido insulinotrópico similar al glucagón tipo 1 (GLP-1), el cual actúa sobre la secreción de las células $\beta$ con una capacidad para aumentar la sensibilidad de la glucosa en las células $\beta$, lo que contribuye a la mejoría de ese parámetro ${ }^{(9)}$.

Las incretinas son péptidos gastrointestinales secretados en respuesta a la ingesta de alimentos que mejoran la secreción de insulina. Las principales incretinas son el péptido inhibidor gástrico (GIP), secretado por las células K en el intestino delgado proximal, y GLP-1, secretado por las células $\mathrm{L}$ del intestino delgado distal; el GLP-1 retrasa el vaciado gástrico, disminuye el apetito, inhibe el glucagón y favorece la sensibilidad a la insulina. Se ha expuesto que las incretinas intervienen como mediadores clave de los efectos antidiabéticos de ciertos tipos de $\mathrm{CB}^{(10)}$.

Uno de los cambios que induce la $\mathrm{CB}$ es la reducción significativa de la resistencia a la insulina hepática, sucede en pocos días y alcanza aproximadamente el $50 \%$ de los valores preoperatorios después de solo una semana.

Las mejorías específicas de la insulina hepática son evidentes si se determinan mediante el cálculo de la evaluación del modelo homeostático-valor de resistencia a la insulina (HOMA-IR) a partir de los niveles basales de glucosa e insulina. Se ha demostrado que la grasa hepática es un determinante importante de la sensibilidad a la insulina hepática/HOMA-IR, así como del aclaramiento de insulina ${ }^{(6)}$.

En una investigación realizada en adolescentes posoperados de MG o BGYR se encontró que existe una asociación clínica y estadísticamente significativa en la normalización temprana y sostenida del valor de resistencia a la insulina (HOMA-IR) y la insulina en ayuno, como resultado de realizar actividad física de manera regular, mediante el conteo de pasos a través de un StepWatch $^{\text {rw }}$; con un promedio de 4000 a 9000 pasos por día ${ }^{(11)}$.

Se cree que el mejor control glucémico después de BGYR, además de la pérdida de peso corporal y la restricción calórica, puede ser consecuencia de un aumento de la sensibilidad a la insulina hepática y periférica, así como del aumento de la secreción de insulina posprandial como resultado del aumento de glucagón y del GLP-1 ${ }^{(9)}$. Debido a los notables efectos contra la obesidad de los agonistas del receptor de GLP-1 disponible después de la derivación gástrica y la gastrectomía vertical en manga, la acción central del GLP-1 parecía ser el mecanismo clave por el cual estas cirugías bariá- 
tricas suprimen la ingesta de alimentos. Sin embargo, aún no se confirma la función del GLP-1 en modelos animales murinos de BGYR con deficiencia de receptor de GLP-1 y gastrectomía vertical en manga; incluso los niveles circulantes elevados de GLP-1 periférico endógeno pueden no alcanzar estos receptores centrales en cantidades suficientes $^{(1)}$. A pesar de esto, los efectos del GLP-1 sobre la glucosa y el metabolismo ya se están aprovechando en las terapias basadas en los efectos de GLP-1 ${ }^{(6,12)}$.

Además, los cambios en los niveles de incretinas y su efecto después del BGYR en pacientes con obesidad mórbida y DM2 causan que la glucosa oral incremente la secreción de insulina notablemente un mes después del BGYR, con una mejoría significativa del control de la diabetes. La potencia de la liberación de incretinas puede ser específica de los cambios anatómicos del intestino que resultan de la cirugía $B G Y \mathrm{R}^{(10)}$.

Se ha encontrado que la BGYR puede mejorar la secreción de insulina porque los alimentos parcialmente digeridos se encuentran con el intestino delgado más distalmente que antes de la cirugía, lo que lleva a cambios en la secreción de hormonas intestinales, como el GLP-1 y la grelina. Sin embargo, la MG no reordena el tracto gastrointestinal, pero tiene efectos sobre la secreción de insulina similares a los de la BGYR. De hecho, en la MG aumenta la secreción de GLP-1 en los roedores, similar a lo que se observa con $\mathrm{BGYR}^{(13)}$.

\section{MECANISMOS INTESTINALES}

Los mecanismos involucrados que conducen a los efectos benéficos de la CB son complejos e involucran varios órganos y vías de comunicación. No solo está involucrado el eje intestino-cerebro, sino también los ejes intestino-tejido adiposo, intestino-hígado, intestino-páncreas y músculo-intestino.

Los cambios metabólicos inducidos por la cirugía dentro del propio intestino de manera directa o indirecta se relacionan con las señales neuronales sensoriales y hormonales del tracto gastrointestinal ${ }^{(14)}$.

Es probable que el cerebro sea un receptor clave de señales humorales y neurales del intestino alterado y es el orquestador final de la ingesta y preferencia de alimentos modificados. Los cambios en la cantidad y composición de la ingesta de alimentos, a su vez, provocan cambios adicionales en las funciones intestinales y en las señales al cerebro y otros órganos. Las señales circulantes también llegan al hígado, páncreas, tejido adiposo blanco y marrón, y al músculo. Los cambios funcionales en estos órganos determinan en gran medida la utilización del sustrato y el gasto de energía, y generan otro conjunto de señales humorales y neurales al cerebro, al intestino y entre sí. Junto con la información del intestino, determinan cambios en el flujo de salida endócrino y autónomo a todos los órganos relevantes ${ }^{(14)}$.

La readaptación intestinal posoperatoria, así como la respuesta inmunitaria y la adecuación metabólica son cambios que pueden explicar la respuesta adaptativa aguda aumentada al ayuno y los procesos metabólicos ahorradores de glucosa que se observan en el hígado y el músculo después de la $\mathrm{CB}$ tanto en $\mathrm{MG}$ como en $\mathrm{BGYR}^{(2)}$.

De acuerdo con algunas investigaciones recientes, se ha demostrado que, por una parte, en respuesta a la MG se reduce la capacidad de transporte de glucosa y aumenta la cantidad de células que secretan GLP-1; por otra parte, en respuesta a BGYR, el intestino se vuelve hiperplásico, lo que aumenta la cantidad de células secretoras de GLP-1. Ambos mecanismos son concomitantes a una mejoría en la tolerancia de la glucosa después de la cirugía ${ }^{(2)}$.

\section{MECANISMOS DE PÉRDIDA DE PESO: EQUILIBRIO ENERGÉTICO}

La pérdida de peso causa cambios en el apetito y el gasto energético que pueden promover la recuperación de peso ${ }^{(15)}$.

La CB, en la que se deriva la mayor parte del estómago y el duodeno con el uso de una anastomosis gastroyeyunal, generalmente causa una pérdida de peso invariablemente a largo plazo; sin duda, este procedimiento quirúrgico continúa generando respuestas fisiológicas compensatorias normales al déficit energético. Posiblemente, ese efecto no solo es resultado de la restricción gástrica, ya que también involucra una alteración de los factores hormonales intestinales que regulan la conducta alimentaria ${ }^{(16)}$.

Se conoce que la restricción calórica conduce a la disminución de peso y provoca una alteración entre el apetito y las necesidades energéticas, en el que la presión biológica para recuperar el peso perdido se produce en función del aumento del hambre y la reducción del gasto energético que acompañan la pérdida de peso inducida por la dieta ${ }^{(14)}$. Además, continuar viviendo entre ambientes obesogénicos puede promover en las personas con obesidad que han estado en algún tratamiento de pérdida de peso, se restablezca el balance energético positivo y se recupere el peso y la masa grasa 
corporal hacia sus niveles previos a la dieta; ya que, las señales de privación de energía y nutrientes se envían desde la periferia a las redes cerebrales en el hipotálamo y el rombencéfalo, que a través de las neuronas de segundo orden aumentan el hambre y disminuyen el gasto de energía, lo que hace que se deseen más calorías (energy in) de las necesarias (energy out) ${ }^{(17)}$.

La leptina y la insulina disminuyen rápidamente cuando el peso corporal desciende por debajo del punto de intervención más bajo, lo que causa un aumento en la ingesta de alimentos y una reducción del gasto energético; la disminución de la leptina circulante es mucho mayor que la pérdida de grasa corporal y de tejido magro metabólicamente activo que se encuentra en la mayoría de las personas con obesidad, podría proporcionar señales para impulsar la ingesta excesiva de energía, las cuales pueden ser las causantes, entre otras, de la recuperación del peso ${ }^{(17,18)}$.

Los cambios posoperatorios en el metabolismo energético también se han discutido en profundidad, en particular inspirados en estudios con roedores, que generalmente han mostrado un mayor gasto energético después de BGYR ${ }^{(6)}$.

La elevación de los niveles de grelina en el plasma con la pérdida de peso inducida por la dieta es consistente con la hipótesis de que la grelina tiene un papel en la regulación a largo plazo del peso corporal ${ }^{(15)}$.

La grelina representa un caso especial: aumenta el apetito y la ingesta de alimentos, y su secreción posprandial disminuye después de la BGYR. Un análisis estadístico sugiere fuertemente que su disminución puede estar involucrada en los cambios tempranos del apetito; sin embargo, después de algún tiempo, los niveles de grelina vuelven a los niveles preoperatorios, mientras que el apetito y la ingesta de alimentos permanecen bajos. Los más potentes de estos reguladores del apetito pueden ser el péptido YY $_{3-36}$ (PYY) y el GLP-1, los cuales demuestran que inhiben la ingesta de alimentos en personas con sobrepeso ${ }^{(19)}$.

Los niveles de grelina plasmática de 24 horas aumentan en consecuencia a la pérdida de peso inducida por la dieta, lo que sugiere que puede desempeñar un papel en la respuesta adaptativa que limita la cantidad de peso que se puede perder con la dieta; pero los niveles de grelina son anormalmente bajos después de la derivación gástrica, lo que aumenta la posibilidad de que esta cirugía reduzca el peso en parte al suprimir la producción de grelina, además de que la secreción de este péptido puede disminuir después de la BGYR debido a la denervación de las células secretoras de grelina en el fondo del estómago ${ }^{(15)}$.

En algunos estudios se aborda la posibilidad de que la disminución de los niveles de grelina pueda ser en parte responsable de la reducción del hambre después de la BGYR y por la observación de que la pérdida de peso posoperatoria se correlaciona con la magnitud de la disminución de los niveles de grelina circulante en un modelo murino para BGYR ${ }^{(20)}$.

\section{MECANISMO BIOLÓGICO EN CONJUNTO CON EL ESTILO DE VIDA}

La CB impulsa algunos efectos favorables sostenibles sobre el metabolismo además de la pérdida de peso. Estos efectos benéficos conducen a mejorías en la sensibilidad a la insulina, que resuelven la DM2 ${ }^{(21)}$. Además, después de la $\mathrm{CB}$, los pacientes con obesidad logran una mejoría en la función cardiovascular. Se encontró que la pérdida de peso después de la $\mathrm{CB}$ es capaz de disminuir el riesgo de cáncer relacionado con las hormonas en pacientes con obesidad ${ }^{(22)}$. Mantener con éxito el peso perdido es difícil para muchas personas con sobrepeso y obesidad que hacen dieta para perder peso, se estima que solo alrededor del $20 \%$ de las personas que experimentan una pérdida de peso significativa es capaz de mantener el peso perdido ${ }^{(17)}$.

La investigación Swedish Obese Subjects (SOS) de $\mathrm{CB}$ con seguimiento al cambio de peso después de BGYR, banda gástrica ajustable laparoscópica (LAGB) y MG en comparación con la intervención convencional (cambios en los estilos de vida) es el estudio a largo plazo más completo hasta la fecha ${ }^{(13)}$. En este estudio, los pacientes fueron asignados al azar a cirugía por obesidad grave frente a un grupo control. Los pacientes con obesidad grave o mórbida que fueron tratados quirúrgicamente perdieron $28 \mathrm{~kg}$ de peso en comparación con el grupo control, en el que no hubo una pérdida de peso significativa. Los pacientes tratados quirúrgicamente encontraron una notable mejoría metabólica y una disminución del riesgo cardiovascular, así como regulación de la disnea.

Estos resultados muestran que la $\mathrm{CB}$ es un tratamiento con beneficios sostenibles, reduce las complicaciones microvasculares, especialmente en aquellos pacientes que presentan un estado prediabético o DM2 con una mejoría después de la cirugía, incluso antes de que haya una disminución de peso importante ${ }^{(23)}$. El tiempo de actividad física también se incrementó en comparación con el grupo control ${ }^{(23)}$. 
Hay evidencia de que los factores metabólicos pueden coadyuvar sustancialmente a los resultados del tratamiento en respuesta a la pérdida de peso, pero el comportamiento voluntario (elecciones conscientes) frente al determinismo biológico no son mutuamente excluyentes, y se considera que los factores conductuales, metabólicos y ambientes obesogénicos están entrelazados, los cuales plantean obstáculos importantes para el mantenimiento de la pérdida de peso a largo plazo $^{(24,25)}$; es interesante y esperanzador considerar al ejercicio como parte del estilo de vida que puede mejorar el mantenimiento del peso perdido ${ }^{(18)}$.

Estudios sugieren que la $\mathrm{CB}$ podría contribuir a mejorar el metabolismo mediante la regulación epigenética, dado que puede ser una vía relevante en la modulación de la expresión de los genes implicados en la fisiología y el metabolismo en la obesidad humana ${ }^{(26)}$.

En un análisis realizado en sujetos suecos con obesidad que se sometieron a cirugía gástrica y un grupo control tratado de manera convencional, emparejados contemporáneamente, se encontró que los sujetos tratados quirúrgicamente en este estudio tuvieron una mayor pérdida de peso, realizaron más actividad física y disminuyeron su consumo de energía en comparación con los sujetos del grupo control durante un período de 10 años. Además, las tasas de recuperación a 2 y 10 años de todos los factores de riesgo estudiados de hipertrigliceridemia, diabetes e hiperuricemia, excepto la hipercolesterolemia, fueron más favorables en el grupo de cirugía que en el grupo control ${ }^{(24)}$.

Diferentes estudios han sugerido que la pérdida de peso a gran escala mediante la CB causa una disminución de la fuerza muscular dinámica, estática y ninguna mejoría de la capacidad aeróbica, por lo que un programa de ejercicio intenso podría prevenir esta disminución de función metabólica e incluso inducir un aumento en la fuerza de la mayoría de los grupos musculares, adicional a una mejoría en la capacidad aeróbica; en un estudio se demostró el aumento significativo del potencial funcional, y se observaron cambios con respecto a la composición corporal (disminución de la masa grasa y aumento de la masa libre de grasa). También se encontró que la intervención de entrenamiento con ejercicios de fuerza causó efectos positivos con respecto a la pérdida de masa muscular después de la $\mathrm{CB}$, los pacientes sin programa de ejercicio que solo se sometieron a $\mathrm{CB}$ mostraron una disminución de la fuerza muscular y los que estuvieron bajo entrenamiento evitaron este deterioro muscular ${ }^{(27)}$.
Se afirma que el ejercicio tiene efectos favorables para todo el organismo, específicamente en la composición corporal, control de la presión arterial, aumento de la sensibilidad a la insulina, disminución de la inflamación, presencia de biomarcadores de "inflamación" y también aterosclerosis carotídea subclínica, e incluso efectos fisiológicos en el sistema cardiovascular humano, por ejemplo, eliminación de fosfolípidos oxidados de la pared del vaso, estabilización de la aterosclerosis y cambios positivos en el colesterol de baja densidad (LDL). Proponer un programa de entrenamiento físico antes y después de la $\mathrm{CB}$ garantiza un resultado efectivo y duradero, considerando que la condición física no mejora solo con la pérdida de peso; el entrenamiento físico debe ser parte de cada intervención médica o programa de rehabilitación perioperatoria ${ }^{(13,28)}$.

Las conductas compensatorias espontáneas u otros factores ambientales, socioeconómicos y psicológicos afectan las conductas de alimentación y actividad física. Conjuntamente, el metabolismo de un individuo, que está influenciado por la edad, el sexo, la adiposidad, la sensibilidad a la insulina, la capacidad aeróbica intrínseca o la oxidación máxima de grasas durante el ejercicio, también puede moderar el impacto de la actividad física en las adaptaciones homeostáticas y hedónicas que impulsan la recuperación de peso ${ }^{(18)}$.

Se ha comprobado mediante estudios aleatorizados y observacionales en adolescentes que la intervención exhaustiva en el estilo de vida se asocia con una pérdida de peso del $5 \%$ al $8 \%$ y la mejoría de los factores de riesgo cardiovascular; mientras que la $\mathrm{CB}$ se relaciona con una mayor pérdida de peso y una remisión de hipertensión, DM2 y dislipidemia a mediano y largo plazo (1 a 5 años $)^{(21)}$. En un estudio realizado en pacientes adultos que fueron sometidos a CB mediante BGYR o MG se encontró que 5 años después mostraron cambios significativos en términos de control glucémico, con niveles de hemoglobina glucosilada $\left(\mathrm{HbA}_{1 \mathrm{c}}\right)$ de $6,0 \%$ o menos, asociada con las respuestas de GLP-1. La reducción de peso muestra una disminución importante en el IMC, circunferencia de la cintura, disminución del consumo de medicamentos, mejoría en los niveles de triglicéridos, colesterol de alta densidad (HDL) y calidad de vida; y se han encontrado resultados más significativos en los grupos quirúrgicos que en el grupo de terapia médica ${ }^{(16)}$.

Sin embargo, han surgido algunas ventajas del BGYR sobre la MG. A los 5 años, la derivación gástrica se asoció con una mayor pérdida de peso que la MG, con menos medicamentos para la diabetes ${ }^{(29)}$.

Es interesante mencionar que no existe una definición definitiva de éxito quirúrgico o fracaso; sin 
embargo, se sugiere que una pérdida de peso del $50 \%$ en el momento de la cirugía puede representar un éxito quirúrgico. Otros autores sugieren que el éxito quirúrgico debe estar asociado con la clasificación de IMC; es decir, un IMC $<30 \mathrm{~kg} / \mathrm{m}^{2}$ indica un resultado excelente ${ }^{(30)}$, por lo que la CB es una opción favorable en el tratamiento de la obesidad grave. El hecho de que no todos los factores de riesgo asociados con la obesidad mejoraran con la pérdida de peso sostenida subraya la importancia de obtener datos a largo plazo sobre el efecto de la pérdida de peso en la mortalidad general y en las tasas de incidencia de infarto de miocardio, accidente cerebrovascular y cáncer ${ }^{(24)}$.

\section{CONCLUSIONES}

Los diversos mecanismos paralelos que provoca la CB pueden contribuir a su notable capacidad para mantener la mejoría metabólica en comparación con solo realizar dieta hipocalórica o realizar cambios en el estilo de vida, y pueden explicar la razón por la que ha sido la opción de tratamiento más exitosa en todos los sexos, grupos de edad, orígenes étnicos y raciales, puntos de corte del IMC, comorbilidades y antecedentes genéticos.

La CB se ha establecido como la estrategia más eficaz para inducir una pérdida de peso sostenida y mejorar el metabolismo para controlar la obesidad mórbida y sus comorbilidades sistémicas. Estos procedimientos mejoran la longevidad, la calidad de vida y conducen a la remisión de la DM2 y a la reducción del riesgo cardiovascular, a menudo independientemente de la pérdida de peso. Los efectos fisiológicos de la CB incluyen reducción del tamaño gástrico, reordenamiento anatómico del intestino y flujo alterado de nutrientes, manipulación vagal y modulación de la hormona intestinal entérica, con la modulación asociada del microbioma intestinal y una multitud de procesos posteriores, efectos fisiológicos y modificadores de enfermedades metabólicas. Sin embargo, la CB puede generar algunas complicaciones y un porcentaje de los candidatos a dicho tratamiento fracasan durante este proceso.

Es más probable que la BGYR promueva un balance energético negativo al reducir la motivación para comer, mediada al menos en parte, por cambios en GLP-1, PYY y grelina posprandiales.

\section{PUNTOS CLAVE}

- La CB es el tratamiento para la obesidad mórbida más efectivo a largo plazo, pero no está libre de complicaciones.
- La CB ocasiona cambios profundos en la secreción de hormonas intestinales con efectos sobre el metabolismo, apetito e ingesta de alimentos.

- Mejora la tolerancia a la glucosa hepática y periférica, y pérdida de peso, además de que permite un balance energético negativo.

- Aumenta la secreción de insulina posprandial provocada particularmente por respuestas mayores del GLP-1.

- Disminuye la inflamación crónica y mejora el estado metabólico, lo que se traduce en una mayor calidad de vida.

\section{Declaración de relevancia clínica}

La CB es un tratamiento sostenible que impulsa una secreción exagerada de hormonas intestinales reguladoras del apetito, GLP-1 y PYY, por una exposición y absorción aceleradas de nutrientes en el intestino, lo que refiere el éxito a largo plazo del mantenimiento del peso perdido.

\section{Declaración de autoría}

Delgadillo Arteaga y Nava-González contribuyeron igualmente a la concepción y diseño de la investigación; Nava-González contribuyó al diseño de la investigación; Delgadillo Arteaga contribuyó a la adquisición y análisis de los datos; Delgadillo Arteaga y NavaGonzález contribuyeron a la interpretación de los datos y Delgadillo Arteaga y Nava-González redactaron el manuscrito. Todos los autores revisaron el manuscrito, acuerdan ser plenamente responsables de garantizar la integridad y precisión del trabajo, y leyeron y aprobaron el manuscrito final.

\section{Conflictos de interés}

Los autores declaran no tener conflictos de interés.

\section{Fuentes de financiamiento}

El presente estudio no tuvo financiación.

\section{Referencias bibliográficas}

1. Badman MK, Flier JS. The gut and energy balance: visceral allies in the obesity wars. Science. 2005;307(5717):1909-14. doi: 10.1126/science.1109951

2. Cavin JB, Couvelard A, Lebtahi R, Ducroc R, Arapis $\mathrm{K}$, Voitellier $\mathrm{E}$, et al. Differences in Alimentary Glucose Absorption and Intestinal Disposal of Blood Glucose 
After Roux-en-Y Gastric Bypass vs Sleeve Gastrectomy. Gastroenterology. 2016;150(2):454-64.e9. doi: 10.1053/j. gastro.2015.10.009

3. Ziogas IA, Zapsalis K, Giannis D, Tsoulfas G. Metabolic syndrome and liver disease in the era of bariatric surgery: What you need to know! World J Hepatol. 2020;12(10):709-721. doi: 10.4254/wjh.v12.i10.709

4. Angrisani L, Santonicola A, Iovino P, Formisano G, Buchwald H, Scopinaro N. Bariatric Surgery Worldwide 2013. Obes Surg. 2015;25(10):1822-32. doi: 10.1007/s11695-015-1657-z

5. Molin Netto BD, Earthman CP, Farias G, Landi Masquio DC, Grotti Clemente AP, Peixoto P, et al. Eating patterns and food choice as determinant of weight loss and improvement of metabolic profile after RYGB. Nutrition. 2017;33:125-131. doi: 10.1016/j.nut.2016.05.007

6. Holst JJ, Madsbad S, Bojsen-Møller KN, Svane MS, Jørgensen NB, Dirksen C, et al. Mechanisms in bariatric surgery: Gut hormones, diabetes resolution, and weight loss. Surg Obes Relat Dis. 2018;14(5):708-714. doi: 10.1016/j.soard.2018.03.003

7. Sjöström L. Review of the key results from the Swedish Obese Subjects (SOS) trial - a prospective controlled intervention study of bariatric surgery. J Intern Med. 2013;273(3):219-34. doi: $10.1111 /$ joim. 12012

8. Zhang C, Zhang J, Liu Z, Zhou Z. More than an Antidiabetic Bariatric Surgery, Metabolic Surgery Alleviates Systemic and Local Inflammation in Obesity. Obes Surg. 2018;28(11):3658-3668. doi: 10.1007/s11695-018-3400-z

9. Kaska L, Sledzinski T, Chomiczewska A, Dettlaff-Pokora A, Swierczynski J. Improved glucose metabolism following bariatric surgery is associated with increased circulating bile acid concentrations and remodeling of the gut microbiome. World J Gastroenterol. 2016;22(39):8698-8719. doi: 10.3748/wjg. v22.i39.8698

10. Laferrère B, Heshka S, Wang K, Khan Y, McGinty J, Teixeira J, et al. Incretin levels and effect are markedly enhanced 1 month after Roux-en-Y gastric bypass surgery in obese patients with type 2 diabetes. Diabetes Care. 2007;30(7):1709-16. doi: $10.2337 / \mathrm{dc} 06-1549$

11. Price PH, Kaizer AM, Inge TH, Eckel RH. Physical activity impacts insulin sensitivity post metabolic bariatric surgery in adolescents with severe obesity. Int J Obes (Lond). 2020;44(7):1479-1486. doi: 10.1038/s41366-020-0585-8

12. Berthoud HR, Morrison CD, Münzberg H. The obesity epidemic in the face of homeostatic body weight regulation: What went wrong and how can it be fixed? Physiol Behav. 2020;222:112959. doi: 10.1016/j.physbeh.2020.112959

13. Leibel RL, Seeley RJ, Darsow T, Berg EG, Smith SR, Ratner R. Biologic Responses to Weight Loss and Weight Regain: Report From an American Diabetes Association Research Symposium. Diabetes. 2015;64(7):2299-309. doi: 10.2337/ db15-0004

14. Berthoud HR, Shin AC, Zheng H. Obesity surgery and gutbrain communication. Physiol Behav. 2011;105(1):106-19. doi: 10.1016/j.physbeh.2011.01.023
15. Cummings DE, Weigle DS, Frayo RS, Breen PA, Ma MK, Dellinger EP, et al. Plasma ghrelin levels after diet-induced weight loss or gastric bypass surgery. N Engl J Med. 2002;346(21):1623-30. doi: 10.1056/NEJMoa012908

16. Schauer PR, Bhatt DL, Kirwan JP, Wolski K, Aminian A, Brethauer SA, et al. Bariatric Surgery versus Intensive Medical Therapy for Diabetes - 5-Year Outcomes. N Engl J Med. 2017;376(7):641-651. doi: 10.1056/NEJMoa1600869

17. Melby CL, Paris HL, Foright RM, Peth J. Attenuating the Biologic Drive for Weight Regain Following Weight Loss: Must What Goes Down Always Go Back Up? Nutrients. 2017;9(5):468. doi: 10.3390/nu9050468

18. Foright RM, Presby DM, Sherk VD, Kahn D, Checkley LA, Giles ED, et al. Is regular exercise an effective strategy for weight loss maintenance? Physiol Behav. 2018;188:86-93. doi: 10.1016/j.physbeh.2018.01.025

19. Andrew CA, Umashanker D, Aronne LJ, Shukla AP. Intestinal and Gastric Origins for Diabetes Resolution After Bariatric Surgery. Curr Obes Rep. 2018;7(2):139-146. doi: 10.1007/ s13679-018-0302-2

20. Stylopoulos N, Davis P, Pettit JD, Rattner DW, Kaplan LM. Changes in serum ghrelin predict weight loss after Roux-en-Y gastric bypass in rats. Surg Endosc. 2005;19(7):942-6. doi: 10.1007/s00464-004-8825-x

21. Jakobsen GS, Småstuen MC, Sandbu R, Nordstrand N, Hofsø D, Lindberg M, et al. Association of Bariatric Surgery vs Medical Obesity Treatment With Long-term Medical Complications and Obesity-Related Comorbidities. JAMA. 2018;319(3):291-301. doi: 10.1001/jama.2017.21055

22. Mackenzie H, Markar SR, Askari A, Faiz O, Hull M, Purkayastha S, et al. Obesity surgery and risk of cancer. Br J Surg. 2018;105(12):1650-1657. doi: 10.1002/bjs.10914

23. Sjöström L, Narbro K, Sjöström CD, Karason K, Larsson $\mathrm{B}$, Wedel $\mathrm{H}$, et al. Effects of bariatric surgery on mortality in Swedish obese subjects. N Engl J Med. 2007;357(8):741-52. doi: 10.1056/NEJMoa066254

24. Sjöström L, Lindroos AK, Peltonen M, Torgerson J, Bouchard C, Carlsson B, et al. Lifestyle, diabetes, and cardiovascular risk factors 10 years after bariatric surgery. $\mathrm{N}$ Engl J Med. 2004;351(26):2683-93. doi: 10.1056/NEJMoa035622

25. Karason K, Lindroos AK, Stenlöf K, Sjöström L. Relief of cardiorespiratory symptoms and increased physical activity after surgically induced weight loss: results from the Swedish Obese Subjects study. Arch Intern Med. 2000;160(12):1797802. doi: $10.1001 /$ archinte.160.12.1797

26. Sumithran P, Proietto J. The defence of body weight: a physiological basis for weight regain after weight loss. Clin Sci (Lond). 2013;124(4):231-41. doi: 10.1042/CS20120223

27. Stegen S, Derave W, Calders P, Van Laethem C, Pattyn P. Physical fitness in morbidly obese patients: effect of gastric bypass surgery and exercise training. Obes Surg. 2011;21(1):61-70. doi: 10.1007/s11695-009-0045-y 
28. Pouwels S, Sanches EE, Cagiltay E, Severin R, Philips SA. Perioperative Exercise Therapy in Bariatric Surgery: Improving Patient Outcomes. Diabetes Metab Syndr Obes. 2020;13:1813-1823. doi: 10.2147/DMSO.S215157

29. van Dijk SJ, Molloy PL, Varinli H, Morrison JL, Muhlhausler BS; Members of EpiSCOPE. Epigenetics and human obe- sity. Int J Obes (Lond). 2015;39(1):85-97. doi: 10.1038/ ijo.2014.34

30. da Cruz MRR, Branco-Filho AJ, Zaparolli MR, Wagner NF, de Paula Pinto JS, Campos ACL, et al. Predictors of Success in Bariatric Surgery: the Role of BMI and Pre-operative Comorbidities. Obes Surg. 2018;28(5):1335-1341. doi: $10.1007 / \mathrm{s} 11695-017-3011-0$ 